\title{
Dynamical Hysteresis and Thermal Effects in Vertical-Cavity Surface-Emitting Lasers
}

\author{
Maria Susana Torre and Cristina Masoller
}

\begin{abstract}
We study numerically the polarization-resolved light-output characteristic of vertical-cavity surface-emitting lasers (VCSELs) using the spin-flip model for VCSELs extended to take into account thermal effects via a dynamical equation for the active region temperature, including heat dissipation, heating due to nonradiative carrier recombination, and Joule effect. The temperature dynamics is coupled to the carrier and optical field dynamics via a frequency and temperaturedependent gain coefficient. We show that the interplay of thermal effects and injection current variation can result in turn-on and turn-off hysteresis cycles that can be, depending on various model parameters, positive or negative. In the first case, the turn-on occurs at a higher value of the bias current than the turn-off; in a negative hysteresis cycle, the laser turns-on at a lower value of the bias current than the turn-off. These results are interpreted in terms of the interplay of the two time-scales determined by the injection current swept rate and the thermal relaxation rate.
\end{abstract}

Index Terms-Hysteresis, polarization switching, semiconductor lasers, thermal effects, vertical-cavity surface-emitting lasers (VCSELs).

\section{INTRODUCTION}

$\mathbf{L}$ ONG wavelength vertical-cavity surface-emitting lasers (VCSELs) are nowdays cost-efficient light sources for optical fiber communication systems [1]. Great advantages of these lasers are their very low lasing threshold under continuous-wave room temperature (RT) operation, their highmodulation bandwidth and high-coupling efficiency into optical fibers [2]. Monolithic, 1-D and 2-D VCSEL arrays, individually addressable, can be fabricated by lithography. InP-based devices, emitting at telecommunication wavelengths around $1.3 \mu \mathrm{m}$ and $1.55 \mu \mathrm{m}$ and showing modulation bandwidth in excess of $10 \mathrm{GHz}$ over a wide temperature range have been recently demonstrated [3], [4].

Manuscript received November 17, 2009; revised February 5, 2010; accepted March 15, 2010. Date of current version November 24, 2010. This work was supported by the U.S. Air Force Office of Scientific Research, under Grant FA9550-07-1-0238. The work of C. Masoller was supported by the Spanish Ministerio de Educacion y Ciencia, under Projects FIS2008-06024C03-0 and FIS2009-13360-C03-02. The work of M. S. Torre was supported by the National Council Research, Argentina, under Grant PIP 114-20080100163. This paper was recommended by Associate Editor L. J. Mawst.

M. S. Torre is with the Instituto de Física Arroyo Seco, Facultad de Ciencias Exactas, Universidad Nacional del Centro de la Provincia de Buenos Aires (UNCPBA), Tandil 7000, Argentina (e-mail: marita@exa.unicen.edu.ar).

C. Masoller is with the Departament de Física i Enginyeria Nuclear, Universitat Politecnica de Catalunya, Terrassa 08222, Spain (e-mail: cristina.masoller@upc.edu).

Color versions of one or more of the figures in this paper are available online at http://ieeexplore.ieee.org.

Digital Object Identifier 10.1109/JQE.2010.2046139
In contrast to $850 \mathrm{~nm}$ VCSELs, that are designed such that the gain peak and the cavity resonance are nearly aligned at RT long-wavelength VCSELs have a relatively large spectral detuning between the gain peak and the cavity resonance at RT, which results in enhanced threshold temperature sensitivity [5]. Additionally, for large-scale VCSEL arrays, thermal crosstalk via the heat sink can be an important problem.

Stable wavelength operation and high efficiency are important for applications; however, the wavelength temperature redshift and the decrease of output power and efficiency due to self-heating at high-injection current densities are well known; the latter being particularly relevant in small size devices [6].

Another VCSEL drawback are polarization instabilities that arise mainly because of the cavity circular transverse geometry, which results in weak anisotropies determining the polarization of the laser output. While some devices emit a stable polarization over the entire bias current operating range, others exhibit a variety of polarization regimes, and either the two linearly orthogonal polarization modes are active at the same time, or only one mode is active.

Because the linear orthogonal polarizations are spectrally split by the material birefringence (the splitting typically being of the order of few tens of GHzs), they also have slightly different material gains. Since the cavity wavelength temperature change is relatively small compared with that of the gain peak (of the order of $0.08 \mathrm{~nm} /{ }^{\circ} \mathrm{C}$ and $0.34 \mathrm{~nm} /{ }^{\circ} \mathrm{C}$, respectively), as the bias current increases, device heating can result in a change of sign of the gain-to-loss ratio of the two polarizations which can in turn result in a polarization switch (PS) [7].

A nonthermal mechanism for explaining the PS was proposed within the context of the spin-flip model (SFM) [8], [9]. In this model, the linear orthogonal polarizations are associated with phase-locked states of the two circularly polarized components of the optical field, with a phase difference equal either to 0 or to $\pi$. The initial polarization preferred at threshold is determined by the difference in gain-to-loss ratios; however, as the injection current is increased, this difference can be overcome by a change of stability of the phase locked states, resulting in a PS. An extension of the SFM model including an explicit form of a frequency-dependent complex susceptibility of the uniaxially stressed quantum well semiconductor material and thermal shift of the gain curve was proposed in [10], and the model predictions were compared with experiments on an air-post VCSEL operating at $980 \mathrm{mn}$. 
TABLE I

PARAMETER VALUES

\begin{tabular}{|l|c|}
\hline Value & Parameter \\
\hline$k=41.7 \mathrm{~ns}^{-1}$ & Field decay rate \\
$\gamma_{N}=2 \mathrm{~ns}^{-1}$ & Carrier decay rate \\
$\gamma_{j, 0}=100 \mathrm{~ns}^{-1}$ & Spin-flip decay rate at RT \\
$\gamma_{T}=0.01 \mathrm{~ns}^{-1}$ & Temperature decay rate \\
$\delta_{0}=0 \mathrm{~nm}$ & RT gain-cavity offset \\
$\gamma_{a}=0.4 \mathrm{~ns}^{-1}, \gamma_{p}=60 \mathrm{rad} / \mathrm{ns}$ & Anisotropy parameters \\
$\alpha=3$ & Alpha-factor \\
$\beta_{s p}=10^{-4} \mathrm{~ns}^{-1}$ & Spontaneous emission noise \\
$K=7.5$ & Dimensionless parameter \\
$Z=0.32{ }^{\circ} \mathrm{C} / \mathrm{ns}$ & Carrier heating coefficient \\
$P=3.69 \times 10^{-3}{ }^{\circ} \mathrm{C} / \mathrm{ns}$ & Joule heating coefficient \\
\hline
\end{tabular}

Recently, we proposed an extension of the SFM model [11] incorporating a rate-equation for the variation of the temperature of the active region, which takes into account the decay to a fixed substrate temperature, Joule heating, and nonradiative recombination heating [12], [13]. The extended SFM model also takes into account a frequency and temperature-dependent material gain, and the red shift of the gain peak and of the cavity resonance with increasing temperature; it is therefore suitable for studying the interplay of polarization and thermal effects.

In this paper, we employ this model to compute the polarization-resolved light-output (LI) curve of a VCSEL and to analyze the influence of various model parameters. In particular, we consider the effect of the speed of the ramp signal used to scan the bias current. We show that the interplay of thermal effects and current variation can result in turn-on and turn-off hysteresis cycles that can be, depending on model parameters, positive or negative. In the first case, the turn-on occurs at a higher value of the injection current than the turnoff; in a negative hysteresis cycle, the laser turns-on at a lower value of the injection current than the turn-off.

This paper is organized as follows. Section II presents the extended SFM model, Section III presents the numerical results, and Section IV presents a summary and the conclusion.

\section{MODEL}

The rate equations of the extended SFM model are [11]

$$
\begin{aligned}
\frac{d E_{ \pm}}{d t}= & k(1+i \alpha)\left[g\left(\omega_{ \pm}, T\right) N_{ \pm}-1\right] E_{ \pm} \\
& -\left(\gamma_{a}+i \gamma_{p}\right) E_{\mp}+\sqrt{\beta_{s p}} \xi_{ \pm}(t) \\
\frac{d N_{ \pm}}{d t}= & -\gamma_{N}\left[N_{ \pm}-\mu+2 g\left(\omega_{ \pm}, T\right) N_{ \pm}\left|E_{ \pm}\right|^{2}\right] \\
& -\gamma_{j}\left(N_{ \pm}-N_{\mp}\right) \\
\frac{d T}{d t}= & -\gamma_{T}\left(T-T_{s}\right)+Z(N / K+1)+P\left(\mathcal{I} / \mathcal{I}_{0}\right)^{2} .
\end{aligned}
$$

Here, $E_{+}$and $E_{-}$are the slowly-varying complex amplitudes of the two circularly polarized components of the optical field, $\omega_{+}$and $\omega_{-}$are their angular frequencies, which are calculated self-consistently as $\omega_{ \pm}=\operatorname{Im}\left[\dot{E}_{ \pm} / E_{ \pm}\right], N_{+}$and $N_{-}$ are two carrier populations that have opposite spin orientation, $N=\left(N_{+}+N_{-}\right) / 2=K\left(\mathcal{N} / \mathcal{N}_{0}-1\right)$, where $K$ is a dimensionless

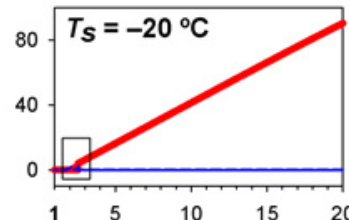

(a)

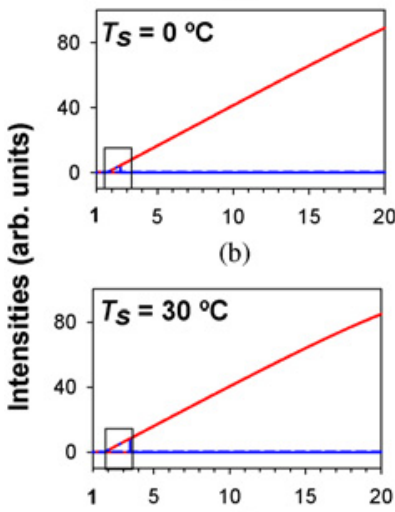

(c)

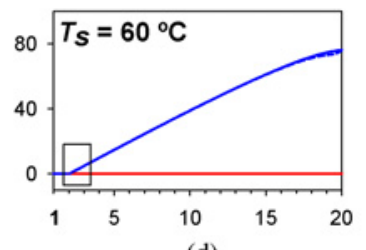

(d)

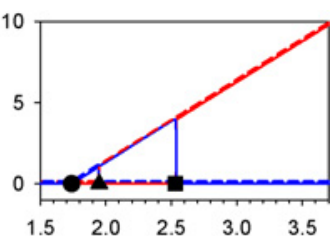

(e)

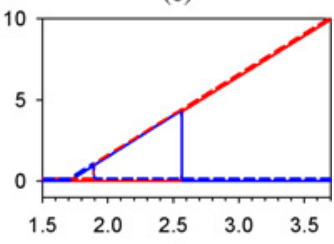

(f)

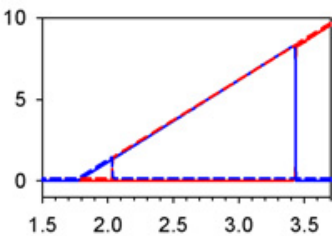

(g)

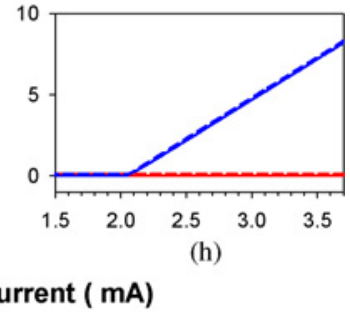

Fig. 1. Polarization-resolved output intensity as a function of the bias current, for various values of the substrate temperature, $T_{s}$. The gain-cavity offset at RT is $\delta_{0}=-3 \mathrm{~nm}$, the duration of the current modulation cycle is $\Delta T=50 \mu \mathrm{s}$, and other parameters are as in Table I. (a)-(d) Whole range of variation of the bias current. (e)-(h) Detail of the threshold region (indicated by a rectangle in the left column). The solid lines represent the modal intensities for increasing current $[x$ polarization: black (red online), $y$ polarization: gray (blue online)] and the dashed lines represent the modal intensities for decreasing current. Filled symbols indicate $I_{\mathrm{PS}, 1}$ (square), $I_{\mathrm{PS}, 2}$ (triangle) and $I_{\mathrm{th}, \mathrm{s}}(\mathrm{dot})$.

constant, $\mathcal{N}$ is the carrier density and $\mathcal{N}_{0}$ is its value at transparency. $g$ is the gain coefficient, which is frequency and temperature dependent. $\mu$ is the injection current parameter, $\mu=K\left(\mathcal{I} / \mathcal{I}_{0}-1\right)$, where $\mathcal{I}$ is the bias current and $\mathcal{I}_{0}$ is the current needed to reach transparency. $T$ is the temperature of the active region, $T_{s}$ is the substrate temperature, $Z$ and $P$ are parameters representing nonradiative recombination heating and Joule heating, respectively.

The model has four decay rates: 1) the field decay rate, $k$; 2) the carried decay rate, $\left.\gamma_{N} ; 3\right)$ the temperature decay rate, $\gamma_{T}$; and 4) the spin-flip decay rate, $\gamma_{j}$. The latter is assumed to increase linearly with temperature as

$$
\gamma_{j}=\gamma_{j, 0} T / T_{0}
$$

where $T_{0}$ is a reference temperature chosen to be equal to the RT and $\gamma_{j, 0}$ is the spin-flip rate at RT.

Other parameters are: $\alpha$ is the linewidth enhancement factor; $\gamma_{a}$ and $\gamma_{p}$ are parameters representing linear anisotropies, dichroism, and birefringence, respectively, $\beta_{s p}$ is the 


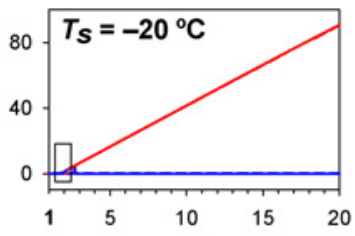

(a)

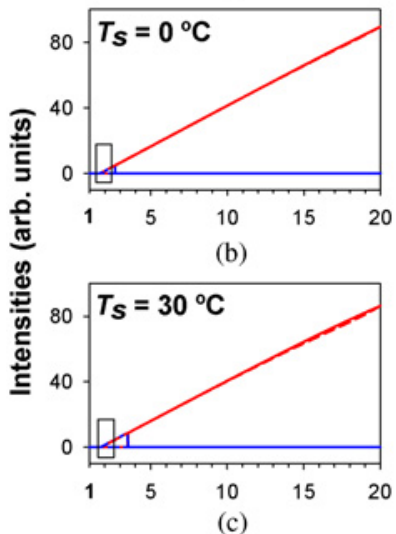

(c)

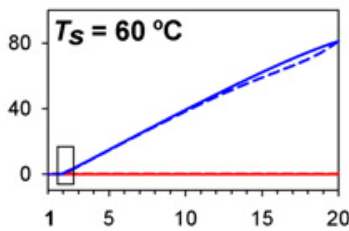

(d)

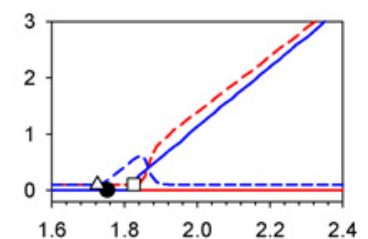

(e)

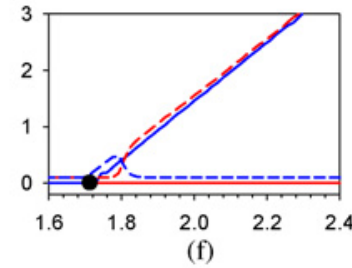

(f)

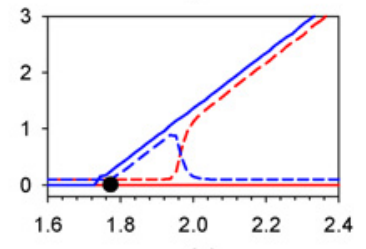

(g)

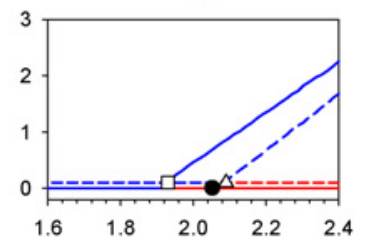

(h)
Injection Current ( $\mathrm{mA}$ )

Fig. 2. Polarization-resolved LI curve for a modulation cycle of $\Delta T=5 \mu \mathrm{s}$, all other parameters as in Fig. 1. (a)-(d) Whole range of variation of the bias current. (e)-(h) Detail of the threshold region. In the right column, the modal intensities for decreasing current (dashed lines) are displaced vertically for clarity. (e)-(h) Dot indicates the value of $I_{\mathrm{th}, \mathrm{s}}$ and empty symbols indicate $I_{\text {on }}$ (square) and $I_{\text {off }}$ (triangle). A hysteresis cycle in the turn-on and turn-off points can be observed, which is such that, at low-substrate temperature [(e) and (f)] $I_{\text {on }}>I_{\text {off }}$ (normal hysteresis); at high-substrate temperature [(g) and (h)] $I_{\text {on }}<I_{\text {off }}$ (negative hysteresis).

spontaneous emission rate and $\xi_{ \pm}$are uncorrelated Gaussian white noises.

The gain coefficient, $g$, which is equal to 1 in the original SFM model [8], is assumed to be Lorentzian in the frequency space with the gain peak and the gain bandwidth decreasing and increasing with temperature, respectively

$$
g(\omega, T)=\frac{T_{0} / T}{1+(\delta-\omega)^{2} /\left[\Delta \omega_{g, 0}^{2}\left(T / T_{0}\right)\right]}
$$

where $\delta(T)=\omega_{g}(T)-\omega_{c}(T)$ is the detuning of the gain peak at $\omega_{g}$ from the cavity mode at $\omega_{c}$, both temperature-dependent, and $\Delta \omega_{g, 0}=2 \pi \times 4 \mathrm{THz}$ is the gain bandwidth at RT $T_{0}$ [14]. The frequency of the gain peak redshifts with the active region temperature as

$$
\hbar \omega_{g}(T)=\varepsilon_{g}^{0}-\alpha^{\prime} T^{2} /(T+\beta)
$$

where $\varepsilon_{g}^{0}=1.52 \mathrm{eV}, \alpha^{\prime}=5.405 \times 10^{-4} \mathrm{eV} / \mathrm{K}$, and $\beta=204 \mathrm{~K}$ are typical values for GaAs-based VCSELs [13]. The cavity mode frequency redshifts with temperature as

$$
\omega_{c}(T)=\left(2 \pi c / \lambda_{0}\right)\left[1-\left(1 / \eta_{0}\right)(d \eta / d T)\left(T-T_{0}\right)\right]
$$

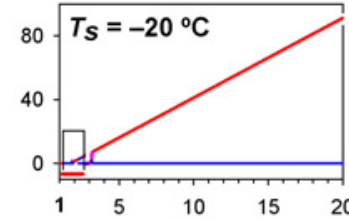

(a)

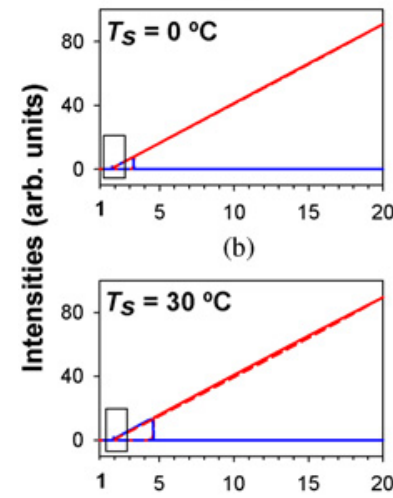

(c)

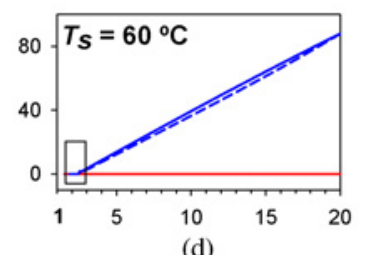

(d)

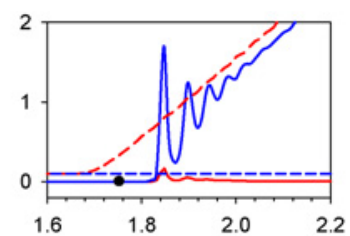

(e)

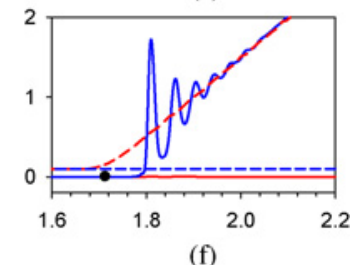

(f)

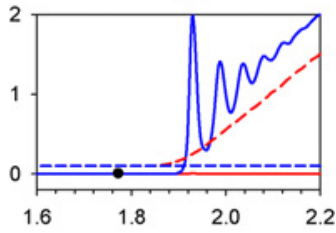

(g)

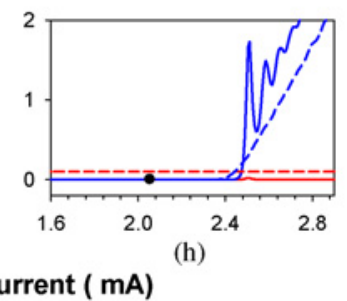

Fig. 3. Polarization-resolved LI curve for a current modulation cycle of $\Delta T=0.5 \mu \mathrm{s}$, all other parameters as in Figs. 1 and 2. (a)-(d) Whole range of variation of the bias current. (e)-(h) Detail of the threshold region. In the right column, the modal intensities for decreasing current (dashed lines) are displaced vertically for clarity. (e)-(g) Dots indicate the value of the static threshold, $I_{\text {th, s. }}$. (e) and (f) There is normal hysteresis, $I_{\text {on }}>I_{\text {off }}$. (g) and (h) There is no hysteresis $\left(I_{\text {on }} \cong I_{\text {off }}\right)$.

where $\lambda_{0}$ and $\eta_{0}$ are the wavelength and the refractive index at the reference temperature $T_{0}$ and $d \eta / d T$ is the rate of variation of the refractive index with temperature. Typical values for VCSELs emitting at $\lambda_{0}=850 \mathrm{~nm}$ are $\eta_{0}=3.41$ and $d \eta / d T=2.8 \times 10^{-4} \mathrm{~K}^{-1}[15]$, which correspond to wavelength variations of $d \lambda_{g} / d T \cong 0.27 \mathrm{~nm} / \mathrm{K}$ and $d \lambda_{c} / d T \cong 0.07 \mathrm{~nm} / \mathrm{K}$ at $T_{0}=300 \mathrm{~K}$.

\section{RESULTS}

We integrated the model equations using, unless otherwise explicitly stated, the parameter values summarized in Table I, which are typical values for a VCSEL of radius $R_{a}=5 \mu \mathrm{m}$ emitting at $\lambda_{0}=850 \mathrm{~nm}$ at RT [16]. An important parameter is the spin-flip rate, $\gamma_{j}$, which affects the dynamics of the orthogonal polarizations, and which is expected to depend strongly on the active region temperature (values employed in the literatures vary in a wide range: $\gamma_{j}=10-1000 \mathrm{~ns}^{-1}$ [17]).

For simplicity here we assume that $\gamma_{j}$ varies linearly with the temperature $\gamma_{j}=\gamma_{j, 0} T / T_{0}$, where $\gamma_{j, 0}=100 \mathrm{~ns}^{-1}$. With this choice there is polarization switching in a wide region of laser parameters, in good agreement with the polarization behavior observed in many VCSELs. In this paper, we focus on studying 


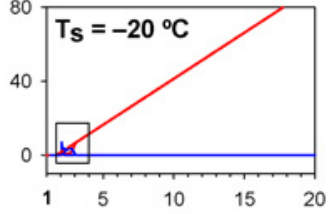

(a)

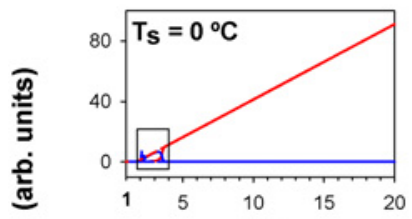

(b)

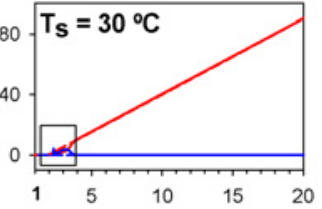

(c)

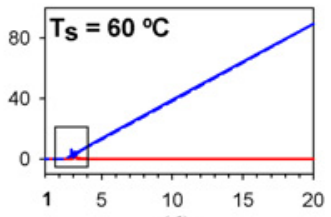

(d)

Injection Current ( $\mathrm{mA})$

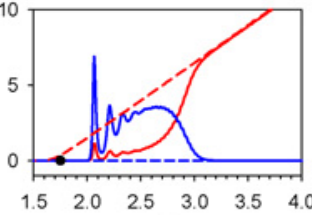

(e)

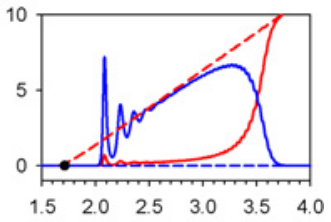

(f)

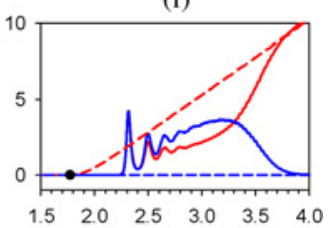

(g)

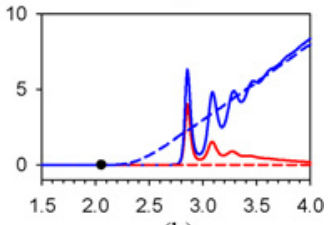

(h) ig. 4. Polarization-resolved LI curve for a current modulation cycle of $\Delta T=0.1 \mu \mathrm{s}$, all other parameters as in Figs. 1-3. (a)-(d) Whole range of variation of the bias current. (e)-(h) Detail of the threshold region. (e)-(g) Dot indicates the value of the static threshold, $I_{\mathrm{th}, \mathrm{s}}$. There is almost no influence of the substrate temperature on the laser turn-on and turn-off points, and only normal hysteresis cycles are observed in (e)-(h).

hysteresis phenomena in the laser switch-on and switch-off and we have verified that the results presented below are robust to other choices of $\gamma_{j, 0}$ : hysteresis in the switch on and switch off points is not affected by the value of $\gamma_{j}$, which affects hysteresis in the polarization of the emitted light, but not in the turn-on and turn-off points.

To compute the LI curve, we simulated the model equations starting with initial conditions at transparency: $E_{+}$and $E_{-}$at the noise level, $\mu=K\left(\mathcal{I} / \mathcal{I}_{0}-1\right)=0, N=K\left(\mathcal{N} / \mathcal{N}_{0}-1\right)=0$, and $T=T_{s}+(Z+P) / \gamma_{T}$. The bias current was increased linearly from the transparency value $\left(\mathcal{I}_{0}=1 \mathrm{~mA}\right)$ to $\mathcal{I}_{\max }=20 \mathrm{~mA}$, then decreased linearly back in a total time interval $\Delta T$, and this process was repeated several times before plotting the resulting LI curve, to disregard transient effects.

Fig. 1 displays the polarization-resolved LI curve, for a bias current modulation period of $\Delta T=50 \mu \mathrm{s}$, and four values of the substrate temperature, $T_{s}$. The modal intensities for increasing (decreasing) current are represented with solid (dashed) lines $[x$ polarization: black (red online), $y$ polarization: gray (blue online)]. The left column displays the whole range of variation of the bias current, while the right column displays a detail of the threshold region.

In Fig. 1, left column, it can be observed that the LI relation is linear, except at high-substrate temperature [Fig. 1(d)], where the characteristic thermal roll-over can be seen. In

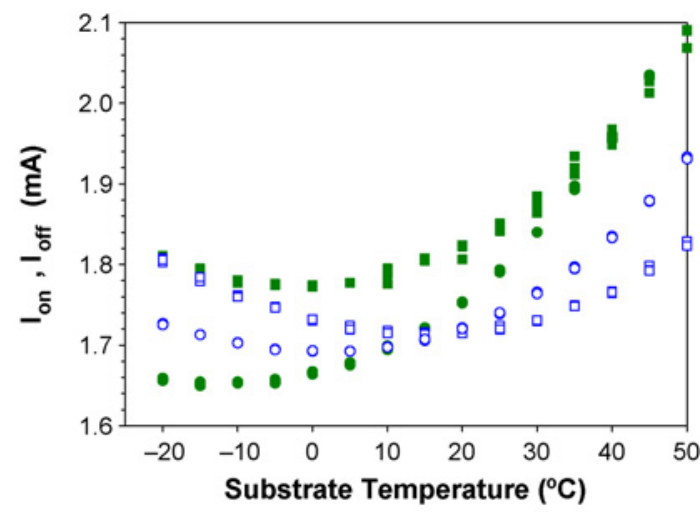

(a)

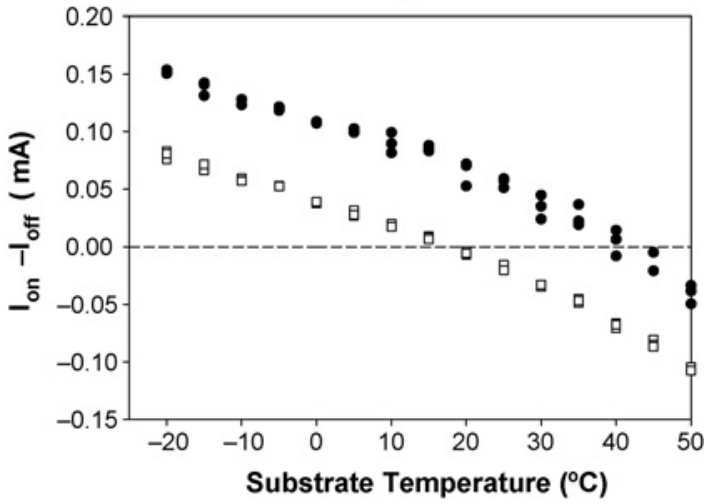

(b)

Fig. 5. (a) Turn-on and turn-off current values, $I_{\text {on }}$ (squares) and $I_{\text {off }}$ (circles) versus the substrate temperature. (b) Size of hysteresis cycle, $I_{\mathrm{on}}-I_{\mathrm{off}}$, versus the substrate temperature. The current modulation cycle is $\Delta T=1 \mu \mathrm{s}$ (filled symbols) and $\Delta T=5 \mu \mathrm{s}$ (empty symbols). We present values for five consecutive modulation cycles to illustrate the dispersion of the turn-on and turn-off points.

Fig. 1, right column, one should notice that there is no hysteresis in the turn-on and turn-off points as they occur both at the same value of the bias current, which is referred to as the static threshold, $I_{\text {th,s }}$. However, in Fig. 1(e)-(g) hysteresis is seen in the polarization switching points for increasing and for decreasing bias current. The PS for increasing current, $I_{\mathrm{PS}, 1}$, occurs at a high-bias current than that for decreasing current, $I_{\mathrm{PS}, 2}$. The variation of the size of the hysteresis cycle with the substrate temperature, and also the fact that in Fig. 1(d) and (h) there is no PS, is due to the fact that the spin-flip rate, $\gamma_{j}$, increases with temperature (4). Since the stability of the $x$ and $y$ polarizations depends strongly on $\gamma_{j}$ [8], it can be expected an enlargement of the PS hysteresis cycle for increasing $T_{s}$, and even the suppression of the PS at high enough temperature.

Fig. 2 displays results for a faster current $\operatorname{ramp}(\Delta T=5 \mu \mathrm{s})$ and all other parameters as in Fig. 1. Dynamic hysteresis in the turn-on and turn-off points can now be observed, as the turnon for increasing current, $I_{\mathrm{on}}$, and the turn-off for decreasing current, $I_{\text {off }}$, do not occur at the same value of the bias current. Moreover, it can be seen that the sign of the hysteresis cycle, $I_{\text {on }}-I_{\text {off }}$, depends on the substrate temperature. For low $T_{s}$ [Fig. 2(e) and (f)], $I_{\text {on }}>I_{\text {off }}$ (we refer to this case as normal or positive hysteresis), while for high $T_{s}$ [Fig. 2(g) and (h)], $I_{\text {on }}<I_{\text {off }}$ (we refer to this case as negative hysteresis). The dots in [Fig. 2(e)-(h)] indicate the value of the static threshold, 


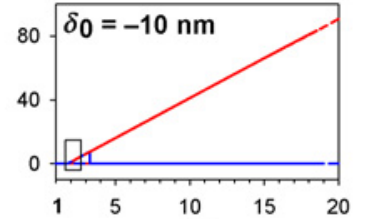

(a)

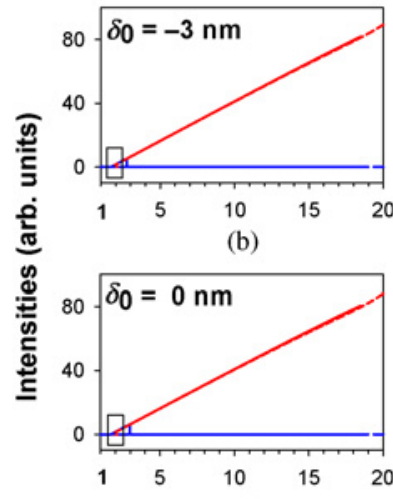

(c)

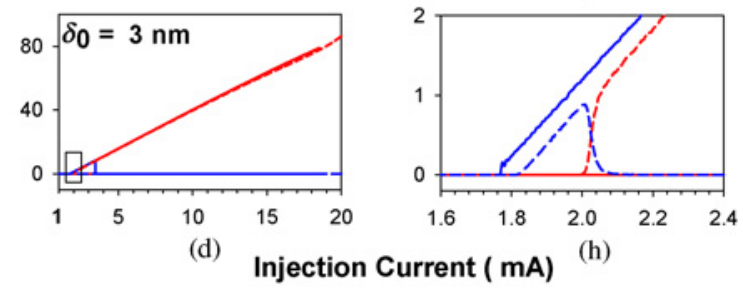

Fig. 6. Polarization-resolved LI curve for a current modulation cycle of $\Delta T=5 \mu \mathrm{s}$, a substrate temperature of $T_{s}=10^{\circ} \mathrm{C}$ and various values of the gain-cavity offset, $\delta_{0}$. Hysteresis in the turn-on and turn-off points is observed and it should be noticed how the hysteresis cycle depends on $\delta_{0}$ : in (e) there is normal hysteresis $\left(I_{\text {on }}>I_{\text {off }}\right)$, in (f) there is no hysteresis $\left(I_{\text {on }} \cong I_{\text {off }}\right)$, and in $(\mathrm{g})$ and $(\mathrm{h})$ there is negative hysteresis $\left(I_{\text {on }}<I_{\text {off }}\right)$. (a)-(d) Whole range of variation of the bias current. (e)-(h) Detail of the threshold region indicated by a rectangle in the left column.

$I_{\mathrm{th}, \mathrm{s}}$, and it can be noticed that in Fig. 2(e) (normal hysteresis), $I_{\mathrm{on}}>I_{\mathrm{th}, \mathrm{s}}$, while in Fig. $2(\mathrm{~g})$ (negative hysteresis), $I_{\mathrm{on}}<I_{\mathrm{th}, \mathrm{s}}$. It should be noticed that in the right column of Fig. 2 we show only a narrow region near threshold (to display the hysteresis on the switch on and switch off points), and thus, the PS for increasing current, which in Fig. 2(a)-(c) occurs outside this region, $I_{\mathrm{PS}, 1}>2.4$, is not seen in Fig. 2(e) $-(\mathrm{g})$. That is why in Fig. 2(e) $-(\mathrm{g})$ it is observed that the laser emits the $y$ polarization (solid line, blue online) when the current is ramped up, and it emits the $x$ polarization (dashed line, red online) when the current is ramped down.

Fig. 3 displays results for an even faster variation of the injection current $(\Delta T=0.5 \mu \mathrm{s})$ and all other parameters as in Figs. 1 and 2. Now the variation of the bias current is fast enough as to observe the characteristic damped relaxation oscillations. In Fig. 3(g) and (h), there is no hysteresis in the turn-on and turn-off points, as $I_{\text {on }} \cong I_{\text {off }}$, while in Fig. 3(e) and (f), there is normal hysteresis, with $I_{\text {on }}>I_{\text {off }}$. The dots in Fig. 3(e)-(h) indicate the value of $I_{\text {th,s }}$ and it can be noticed that in Fig. 3(e) and (f), where there is normal hysteresis, $I_{\text {off }} \cong I_{\text {th }, \mathrm{s}}$ and $I_{\text {on }}>I_{\text {th }, \mathrm{s}}$, while in Fig. $3(\mathrm{~g})$ and $(\mathrm{h})$, where there is no hysteresis, the laser turns on and turns off at $I_{\text {on }}, I_{\text {off }}>I_{\text {th, }, \mathrm{s}}$.

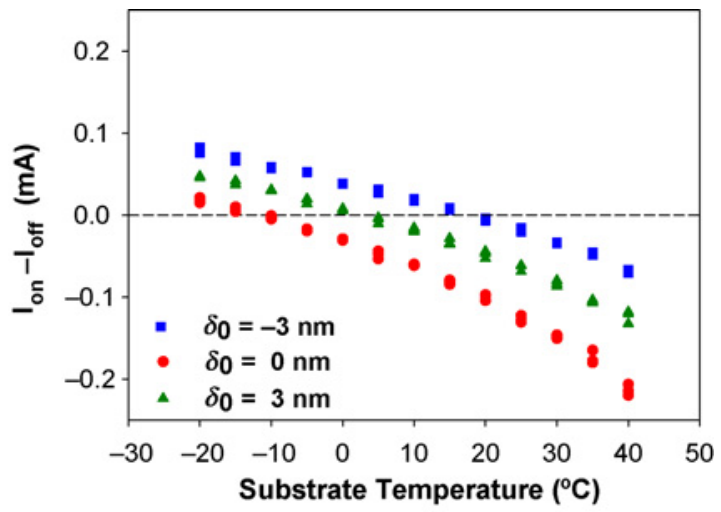

(a)

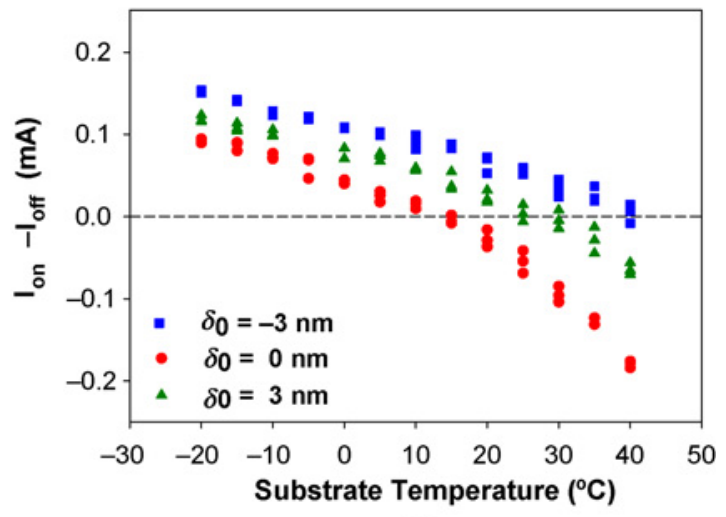

(b)

Fig. 7. Dependence of the hysteresis cycle, $I_{\mathrm{on}}-I_{\mathrm{off}}$ on the substrate temperature, for various values of the gain-cavity offset when the period of the current modulation cycle is (a) $\Delta T=5 \mu \mathrm{s}$ and (b) $\Delta T=1 \mu \mathrm{s}$.

When the bias current is varied even faster, Fig. 4, which is done with $\Delta T=0.1 \mu \mathrm{s}$ and all other parameters are as in Figs. 1-3, shows that thermal effects do not have time to act, and only normal hysteresis cycles are seen for all values of $T_{s}$. The dots in Fig. 4(e)-(h) indicate the value of $I_{\mathrm{th}, \mathrm{s}}$ and in Fig. 4(a)-(h), $I_{\text {off }} \cong I_{\mathrm{th}, \mathrm{s}}$ and $I_{\mathrm{on}}>I_{\mathrm{th}, \mathrm{s}}$

The combined effect of the substrate temperature and the modulation period on the turn-on and turn-off points, and on the size of the hysteresis cycle, $I_{\text {on }}-I_{\text {off }}$, is summarized in Fig. 5(a) and (b), where $I_{\text {on }}$ and $I_{\text {off }}$ [Fig. 5(a)] and $I_{\text {on }}-I_{\text {off }}$ [Fig. 5(b)] are plotted versus the substrate temperature for two values of the current modulation period for which both, normal hysteresis at low $T_{s}$, and negative hysteresis at higher $T_{s}$, are observed $(\Delta T=1 \mu \mathrm{s}$ and $\Delta T=5 \mu \mathrm{s}$, represented with filled and empty symbols, respectively).

As shown in Fig. 6, hysteresis in the turn-on and turnoff points is also observed when the gain-cavity offset, $\delta_{0}=$ $\lambda_{g}\left(T_{0}\right)-\lambda_{c}\left(T_{0}\right)$ is varied, while the substrate temperature and the modulation period are kept constant. Fig. 7 displays the variation of the size of the hysteresis cycle with $T_{s}$, for various values of $\delta_{0}$.

In this model, the hysteresis in the turn-on and turn-off points is due to the interplay of the variation of the bias current (that results in dynamical hysteresis [18]) and the variation of the active region temperature, that is an additional source of hysteresis, because the temperature is a dynamical variable with its own time scale. To illustrate the interplay of these two 


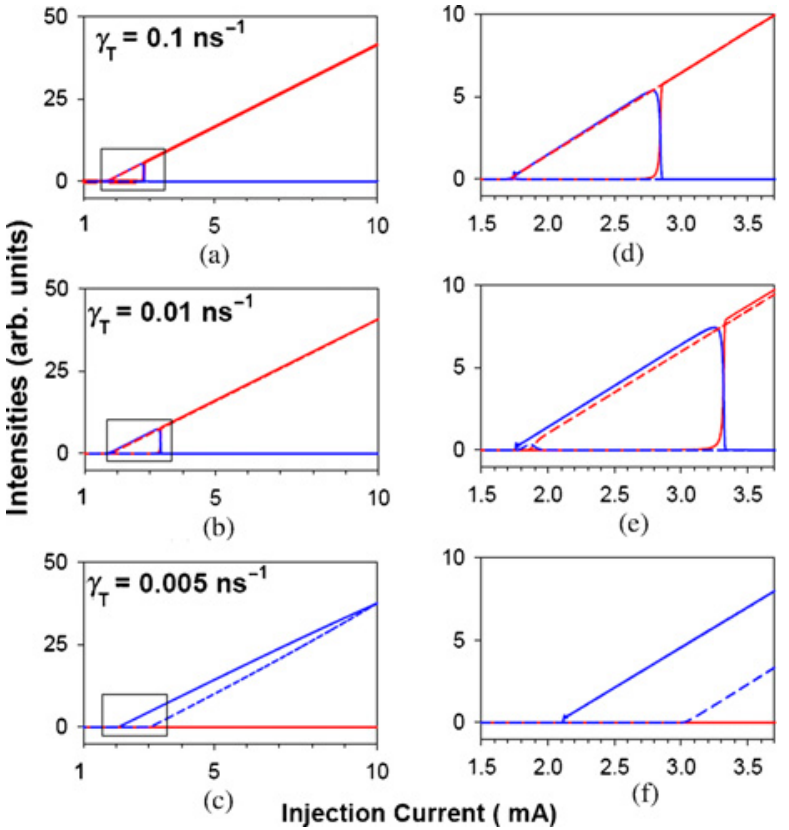

Fig. 8. Polarization-resolved LI curve for a current modulation cycle of $\Delta T=1 \mu \mathrm{s}$, a substrate temperature of $T_{s}=30^{\circ} \mathrm{C}$, a gain-cavity offset of $\delta_{0}=-3 \mathrm{~nm}$ and various values of the temperature decay rate, $\gamma_{T}$. It can be observed that $\gamma_{T}$ significantly affects the hysteresis in the turn-on and turn-off points: almost no hysteresis is observed when the temperature decay rate is fast enough, and on the contrary, large and negative hysteresis cycle is observed when the temperature decay rate is slow enough. (a)-(c) Whole range of variation of the bias current. (d)-(f) Detail of the threshold region indicated by a rectangle in the left column.

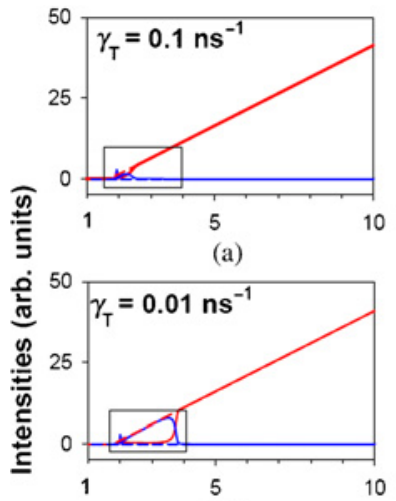

(b)

(c)

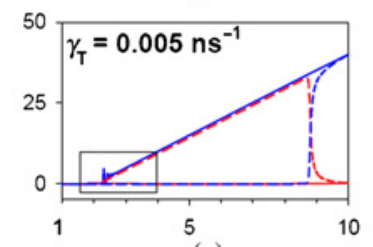

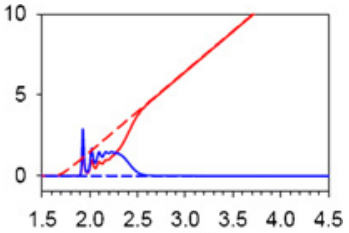

(d)

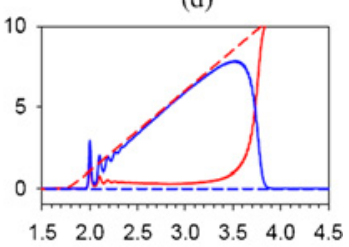

(e)

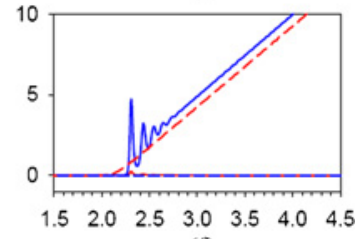

(f)
Fig. 9. As in Fig. 8 but for a faster current $\operatorname{ramp}(\Delta T=0.1 \mu \mathrm{s})$; it can be observed that the value of $\gamma_{T}$ has little influence on the turn-on and turn-off points; however, the dynamics of the two polarizations is different due to the fact that the spin-flip relaxation rate depends on the active region temperature, which in turn depends on $\gamma_{T}$. (a)-(c) Whole range of variation of the bias current. (d)-(f) Detail of the threshold region indicated by a rectangle in the left column. time-scales (bias current variation and temperature variation), in Figs. 8 and 9 we present results keeping $T_{s}$ and $\delta_{0}$ fixed, and changing the temperature decay rate, $\gamma_{T}$, when the current modulation is slow enough as to observe both, normal and negative hysteresis depending on $\gamma_{T}$ (Fig. 8) and when the current variation is fast enough as to observe only normal hysteresis, regardless of the value of $\gamma_{T}$ (Fig. 9). Fig. 8 clearly demonstrates that a slow enough variation of the active region temperature is responsible for the negative hysteresis cycles, while Fig. 9 demonstrates that the time-scale associated to temperature variations does not play any significant role in the turn-on and turn-off points when the bias current is varied fast enough. However, $\gamma_{T}$ still influences the output polarization via the temperature-dependence of the spin-flip rate.

\section{SUMMARY AND CONCLUSION}

We studied numerically the polarization-resolved LI curve of VCSELs using an extension of the SFM that takes into account thermal effects via a dynamical equation for the active region temperature, that includes heat dissipation, heating due to nonradiative carrier recombination, and Joule heating. The temperature dynamics is coupled to the carrier and optical field dynamics via a frequency and temperature-dependent gain coefficient. We showed that the interplay of thermal effects and injection current variation can result in turn-on and turnoff hysteresis cycles that can be, depending on various model parameters, positive or negative. In the first case, the turn-on occurred at a higher value of the bias current than the turn-off; in a negative hysteresis cycle, the VCSEL turns-on at a lower value of the bias current than the turn-off. An important aspect that is not taken into account by the present model is the role of spatial inhomogeneities, i.e., the transverse mode in which the linear polarizations are emitted, the diffusion of carriers and thermal diffusion. The influence of transverse effects is an interesting open question that is left for future work.

While negative hysteresis has been previously observed in a $\mathrm{CO}_{2}$ laser with modulated losses [19], [20], in a semiconductor laser with modulated losses [21], in an injection-locked semiconductor laser [22] and in a passive semiconductor microresonator [23], to the best of our knowledge this is the first report of thermally-induced negative hysteresis in a VCSEL lasing threshold, and we hope that our numerical results will stimulate experimental research into this very interesting phenomenon.

\section{REFERENCES}

[1] C. Lei and J. K. Guenter, Eds., "Vertical-cavity surface-emitting lasers XII," in Proc. SPIE, vol. 6908. 2008.

[2] Y. Suematsu and K. Iga, "Semiconductor lasers in photonics," J. Light. Tech., vol. 26, no. 9, pp. 1132-1144, 2008.

[3] W. Hofmann, E. Wong, M. Ortsiefer, M. Gorblich, G. Bohm, M.-C. Amann, "1-D and 2-D long-wavelength VCSEL arrays for WDM applications and optical interconnects," in Proc. IEEE 21st Int. Semicond. Laser Conf., 2008, pp. 165-166.

[4] M. C. Amann and W. Hofmann, "InP-based long-wavelength VCSELs and VCSEL arrays," IEEE J. Sel. Top. Quantum Electron., vol. 15, no. 3, pp. 861-868, May-Jun. 2009. 
[5] S. Mogg, N. Chitica, U. Christiansson, R. Schatz, P. Sundgren, C. Asplund, and M. Hammar, "Temperature sensitivity of the threshold current of long-wavelength InGaAs-GaAs VCSELs with large gaincavity detuning," IEEE J. Quantum Electron., vol. 40, no. 5, pp. 453462, May 2004

[6] C. Degen, I. Fischer, and W. Elsässer, "Transverse modes in oxide confined VCSELs: Influence of pump profile, spatial hole burning, and thermal effects," Opt. Exp., vol. 5, no. 3, pp. 38-47, 1999.

[7] K. D. Choquette, R. P. Schneider, K. L. Lear, and R. E. Leibenguth, "Gain-dependent polarization properties of vertical-cavity lasers," IEEE J. Sel. Top. Quantum Electron., vol. 1, no. 2, pp. 661-666, Jun. 1995.

[8] J. Martin-Regalado, F. Prati, M. S. Miguel, and N. B. Abraham, "Polarization properties of vertical-cavity surface-emitting lasers," IEEE J. Quantum Electron., vol. 33, no. 5, pp. 765-783, May 1997.

[9] S. Balle, E. Tolkachova, M. San Miguel, J. R. Tredicce, J. MartinRegalado, and A. Gahl, "Mechanisms of polarization switching in single-transverse-mode vertical-cavity surface-emitting lasers: Therma shift and nonlinear semiconductor dynamics," Opt. Lett., vol. 24, no. 16, pp. 1121-1123, 1999.

[10] G. Van der Sande, M. Peeters, I. Veretennicoff, J. Danckaert, G Verschaffelt, and S. Balle, "The effects of stress, temperature, and spin flips on polarization switching in vertical-cavity surface-emitting lasers," IEEE J. Quantum Electron., vol. 42, no. 9, pp. 898-906, Sep.-Oct. 2002.

[11] C. Masoller and M. S. Torre, "Modeling thermal effects and polarization competition in vertical-cavity surface-emitting lasers," Opt. Exp., vol. 16, pp. 21282-21296, Dec. 2008.

[12] T. Rössler, R. A. Indik, G. K. Harkness, J. V. Moloney, and C. Z. Ning, "Modeling the interplay of thermal effects and transverse mode behavior in native-oxide-confined vertical-cavity surface-emitting lasers," Phys. Rev. A, vol. 58, no. 4, pp. 3279-3292, Oct. 1998.

[13] L. Spinelli, G. Tissoni, L. A. Lugiato, and M. Brambilla, "Thermal effects and transverse structures in semiconductor microcavities with population inversion," Phys. Rev. A, vol. 66, no. 2, p. 023817, Aug. 2002.

[14] P. V. Mena, J. J. Morikuni, S.-M. Kang, A. V. Harton, and K. W. Wyat, "A comprehensive circuit-level model of vertical-cavity surface-emitting lasers," J. Light. Technol., vol. 17, no. 12, pp. 2612-2632, Dec. 1999.

[15] J. S. Gustavsson, J. A. Vukusic, J. Bengtsson, and A. Larsson, "A comprehensive model for the modal dynamics of vertical-cavity surfaceemitting lasers," IEEE J. Quantum Electron., vol. 38, no. 2, pp. 203-212, Feb. 2002.

[16] S. Barland, P. Spinicelli, G. Giacomelli, and F. Marin, "Measurement of the working parameters of an air-post vertical-cavity surface-emitting laser," IEEE J. Quantum Electron., vol. 41, no. 10, pp. 1235-1243, Oct. 2005.

[17] E. L. Blansett, M. G. Raymer, G. Khitrova, H. M. Gibbs, D. K. Serkland, A. A. Allerman, and K. M. Geib, "Ultrafast polarization dynamics and noise in pulsed vertical-cavity surface-emitting lasers," Opt. Exp., vol. 9, pp. 312-318, Sep. 2001.

[18] C. Masoller, M. S. Torre, and P. Mandel, "Influence of the injection current sweep rate on the polarization switching of vertical-cavity surface emitting lasers," J. Appl. Phys., vol. 99, article 026108, Jan. 2006

[19] D. Bromley, E. J. D'Angelo, H. Grassi, C. Mathis, and J. R. Tredicce, "Anticipation of the switch-off and delay of the switch-on of a laser with a swept parameter," Opt. Commun., vol. 99, pp. 65-70, May 1993.
[20] A. N. Pisarchik, B. F. Kuntsevich, R. Meucci, and E. Allaria, "Negative hysteresis in a laser with modulated parameters," Opt. Commun., vol. 189, pp. 313-319, Mar. 2001.

[21] S. H. Choi, E. White, D. M. Wood, T. Dodson, K. V. Vasavada, and G. Vemuri, "Delayed bifurcations and negative hysteresis in semiconductor lasers: The role of initial conditions," Opt. Commun., vol. 160, pp. 261267, Feb. 1999.

[22] S. Sivaprakasam, D. N. Rao, and R. S. Pandher, "Demonstration of negative hysteresis in an injection-locked diode laser," Opt. Commun., vol. 176, pp. 191-194, Mar. 2000.

[23] I. M. Perrini, G. Tissoni, T. Maggipinto, and M. Brambilla, "Thermal effects and cavity solitons in passive semiconductor microresonators," J. Opt. B: Quantum Semiclass. Opt., vol. 6, pp. S369-S379, May 2004.

Maria Susana Torre received the Licenciada en Fisica and Ph.D. degrees in physics from the Universidad Nacional del Centro de la Provincia de Buenos Aires (UNCPBA), Tandil, Argentina, in 1994.

From 1995 to 1997, she was a Post-Doctoral Fellow with the Department of Photonics Technology, Escuela Técnica Superior de Ingenieros Telecomunicaciones, Universidad Politécnica de Madrid, Madrid, Spain. Her research was in quantum well semiconductor laser modeling. Since 1988, she has been a Member of the Instituto de Física "Arroyo Seco," UNCPBA, and is currently a Research Professor with the Facultad de Ciencias Exactas. Her primary research interests include external driven laser physics. Her current research interests include modeling and dynamics of vertical-cavity surface-emitting lasers, dynamics of semiconductor laser with external optical feedback and diffusion effects in semiconductor lasers.

Dr. Torre is a Member of the National Council Research, Argentina.

Cristina Masoller was born in Montevideo, Uruguay, in 1963. She received the B.S. and M.S. degrees from the Universidad de la Republica, Montevideo, Uruguay, in 1986 and 1991, respectively, and the Ph.D. degree from Bryn Mawr College, Bryn Mawr, PA, in 1999, all in physics. Her Ph.D. thesis was about nonlinear dynamics of semiconductor lasers with optical feedback, directed by Prof. Neal B. Abraham.

From 1991 to 2004, she was an Assistant Professor with the Facultad de Ciencias, Universidad de la Republica. From 2004 to 2009, she was a "Ramon and Cajal" Researcher with the Universitat Politecnica de Catalunya in Terrassa, Barcelona, Spain, and is currently a Professor Agregat with the Departament de Física i Enginyeria Nuclear, Escola Tècnica Superior d'Enginyeries Industrial i Aeronàutica de Terrassa. Her current research interests, in the field nonlinear photonics, have been the dynamics of semiconductor lasers and the influence of time delays (delayed feedback, laser synchronization with delayed coupling, and stochastic effects induced by the interplay of noise and delay). She has also studied related phenomena occurring in other dynamical systems, such as excitability in neuronal models and collective behavior of large arrays of delayed-coupled chaotic units. 